\title{
Switching among Clouds: An Approach to Ensure Cloud Service Portability
}

\author{
Jitesh Tilokani \\ B.Tech, VII Sem (I.T.) \\ Government Engineering College Ajmer
}

\author{
Deepak Gupta \\ Assistant Professor (Dept. of C.E. \& I.T.) \\ Government Engineering College Ajmer
}

\begin{abstract}
The portability of cloud services has been the major issue of concern among the IT industries. The situations like vendorlock-in, lack of standardized data formats and complex service level agreements are still affecting the majority of IT sector from adapting this widely emerging technology. This paper suggests a new way in order to inculcate the portability among the cloud vendors and to maintain the consumer's trust in cloud services by ensuring that consumer is the ultimate owner of the data throughout the services. The paper proposes introduction of reliable third party (mediator) between the cloud service provider and the cloud service consumer to remove the various portability issues encountered while switching among the clouds.
\end{abstract}

\section{General Terms}

Cloud computing, cloud service portability.

\section{Keywords}

Cloud computing, Cloud Service Portability, Service Provider, reliable third party (storage provider), Service Consumer. Switching Clouds

\section{INTRODUCTION}

Cloud computing is one of the most quickly emerging technology in the present IT world. It has become popular among the businesses that see the information technology among their core competencies, demand a flexible computing environment and seek to achieve more predictable costs [1] The cloud computing has provided the new flexibility to the old traditional way of data accessing and processing. The US National Institute for Science and Technology has defined cloud computing as:

"A model for enabling convenient, on demand network access to the shared pool of configurable computing resources (e.g. networks, servers, storage applications and services) that can be rapidly provisioned and released with minimal management effort or service provider interaction [2]."

Cloud service portability is a process of switching from one cloud service provider to another cloud service provider (also known as switching among the clouds). The cloud service portability ensures competition among the service providers and provides the customers with wider options to switch to in case of service dissatisfaction.

However, cloud service portability being a necessity is almost impossible to implement due to the issues like vendor-lock-in, lack of standardized formats and complex service level agreements.

\subsection{Cloud portability issues:}

\subsubsection{Vendor-lock-in:}

The 'data' is the heart of any organization. A 'closed' data format which is used by one cloud service, but may not be available for license or to be technically supported by another cloud service, can lock data into specific cloud platforms and render transfer between competing cloud providers costly or impossible [3].

\subsubsection{Complex Service Level Agreements:}

To mark their existence among the competitive cloud market, the cloud service providers use the complex service level agreements. The use of the proprietary contracts or service level agreements with extensive disclaimers increases the complexity and uncertainty of the cloud services [4]. The use of "data hostage" clauses in combination with arbitration or litigation clauses by service providers can insulate service providers from liability for material breaches and be used to coerce nonbreaching customers into paying hefty termination fees [1].

The outsourcing business experiences the negative impact of this lack of portability, or "vendor lock-in" phenomenon, when it wants to migrate to another cloud computing service provider and is confronted with a data hostage clause in its outsourcing agreement requiring the business to pay applicable termination fee for the data to be returned [5].

\section{RELATED WORK}

Although the cloud service portability has been the keen area of research since the time when cloud computing came into existence, the field still lacks a profound solution to overcome the major problems encountered. To the best of our knowledge, the paper suggests first of its own type solution, overcoming all the disadvantages encountered in other related works produced till date.

The traditional cloud service model defined by IBM's Cloud Computing Reference Architecture (CCRA) consists of the cloud service creator, cloud service provider and the cloud service consumer [6]. The role of the cloud service creator is limited to the production of the cloud services; the rest of the interaction is between the cloud service provider and the cloud service consumer. In case of any material breach the data is locked within the computers of the service provider until any jurisdiction is held. Thus bringing the organizations (service 
consumers) work to standstill. In this paper we introduce a reliable third party (also referred as mediator, storage provider or data handler) between the cloud service provider and the consumer. The reliable third party (storage provider) provides storage platform to various service providers and is neutral to both the service provider and the service consumer, thus preventing the data lock-in situations encountered earlier.

To give boost to the cloud service portability, tech giants introduced TOSCA (Topology \& Orchestration Specification for Cloud Applications). The portable and standardized management of cloud services is enabled through the TOSCA [7]. The TOSCA aims at the use of the standardized data formats by the cloud service providers and to overcome the situations like vendor-lock-in (caused by the use of the closed or proprietary data formats). Although the use of the standardized format makes portability simpler to great extent but it might be difficult to implement with the different platforms used by the different service providers. The solution in this paper overcomes this problem by introducing a common platform to all the service providers. Also the cost of portability is brought to negligible because rather than switching data from one service provider's platform to another, only the charge of the portion of common platform is switched i.e. the portion held by one service provider is allocated to another service provider.

\section{CLOUD SERVICE PORTABILITY SOLUTION}

There are various approaches to ensure the portability of cloud services:

\subsection{Standardization of formats and service level agreements:}

The first and the foremost step required to ensure the cloud service portability is the standardization of the data formats used by the service providers. The TOSCA led by tech giants is the first and foremost step in this direction. The use of the proprietary or closed data formats hinders the transfer of data to other service provider and makes the portability uneconomic or impossible. This type of competitive advantage is not limited to technical means, such as using non-portable data formats, since analogous lock-in effects can also be accomplished, to varying degrees, through service agreements [1]. Thus, there is need of standardization of service agreements with minimum terms and conditions.

\subsection{Introduction of reliable third party (mediator) between the Service provider and Service Consumer:}

\subsubsection{Traditional Cloud Service model:}

The present cloud service model deals with the processing and management of data stored within the systems of the service provider, which is accessed on-demand by the service consumer. Thus, whenever the customer feels to terminate the agreement or switch to another cloud service provider there is chance of data being locked-in. The data being key to any organization brings the organization into immense pressure to compromise.

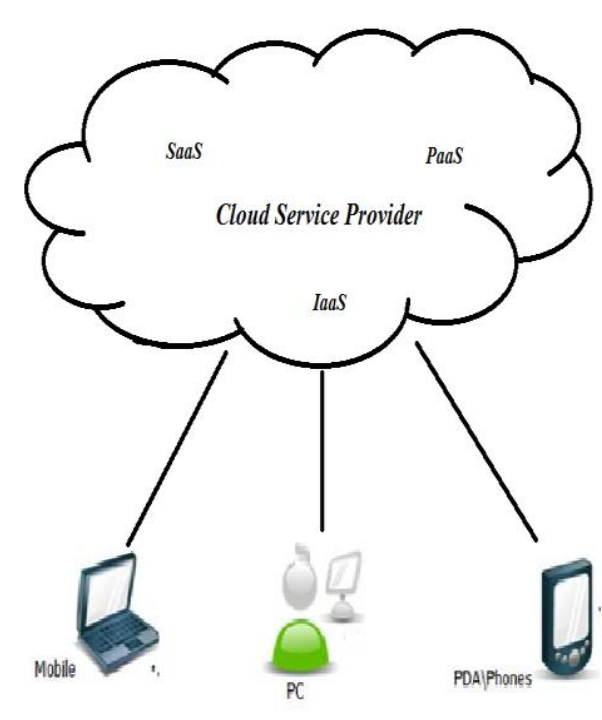

Fig 1: Traditional cloud service model

\subsubsection{Introduction of reliable third party (mediator) between the Service provider and Service Consumer:}

The introduction of the mediator (i.e. reliable third party or storage provider) between the cloud service providers and the service consumers helps to overcome the various portability issues encountered earlier. The storage provider stores the consumers' data and allows the service provider to process and manage the data. The storage provider uses the data format agreed by both the service provider and the consumer. Both the service provider and service consumer have access to the data through secure networks. The introduction of the reliable third party makes the clients data secure and removes the chances of data being mishandled or locked-in in case of termination of service agreement. In case of the termination of the agreement the client just needs to inform the storage provider.

The storage provider then stops the service provider from accessing the client's data. Thus, making the client's data secure and removing the chances of data being mishandled. 


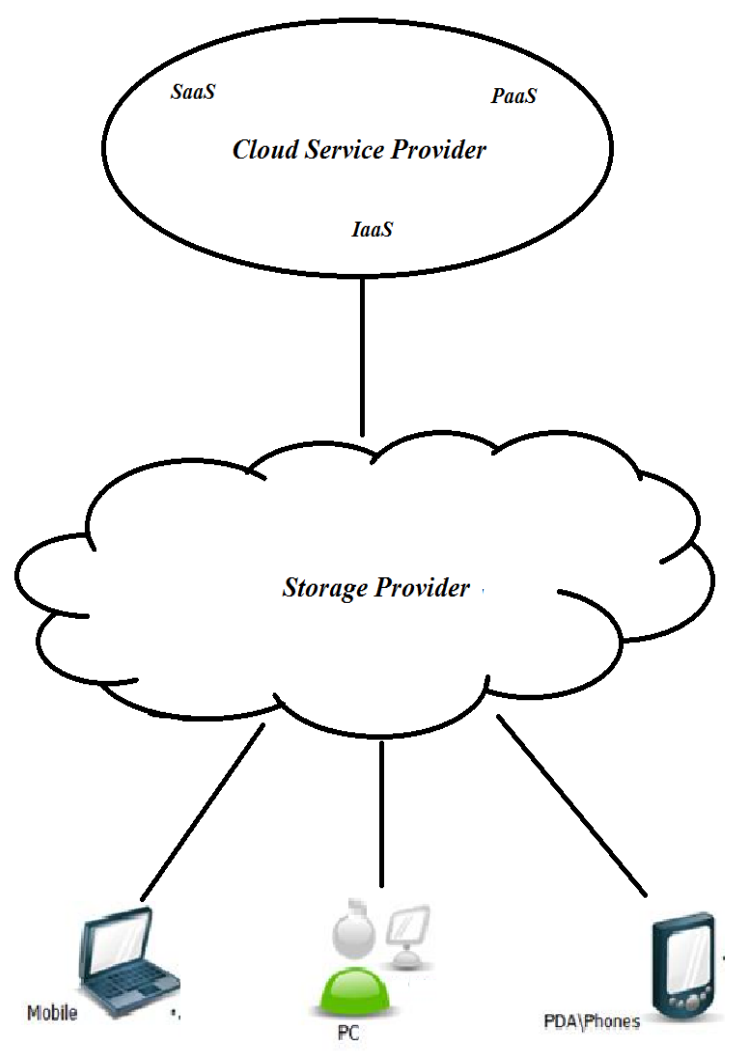

Fig 2: Introduction of mediator (reliable third party) between the service provider and service consumer.

\subsubsection{Introduction of common mediator (common} reliable third party) between various service providers and service consumers:

The introduction of the common mediator (i.e. common reliable third party or storage provider) between various service providers and service consumers can make the portability much simpler, cheaper and faster. By common storage provider, we mean a data storage provider with large storage capacity providing storage platform to various service providers and consumers.

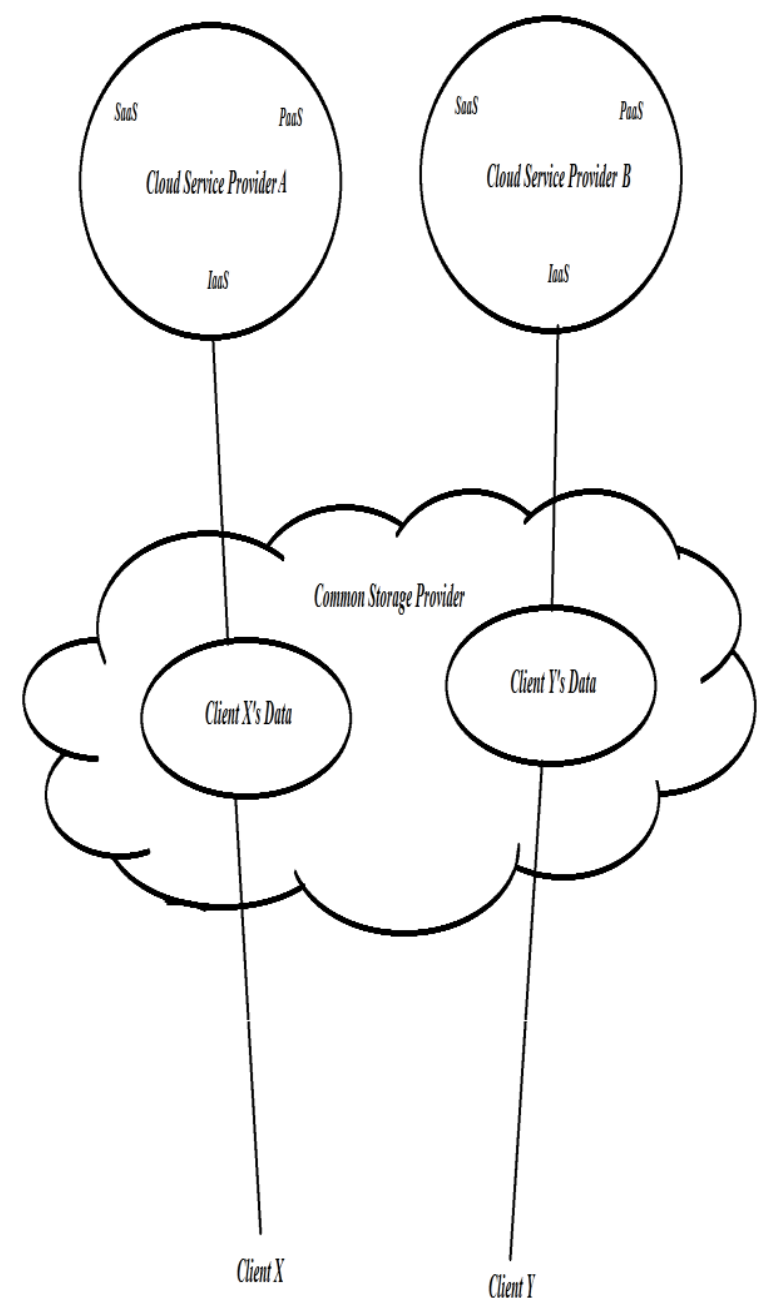

Fig 3: Common mediator (common reliable third party) between various service providers and consumers

The fig 3 depicts a common storage provider, storing data of Client $\mathrm{X}$ and $\mathrm{Y}$. The service providers $\mathrm{A}$ and $\mathrm{B}$ have access to the data of the Client $\mathrm{X}$ and $\mathrm{Y}$, respectively. The security is maintained with the help of partitioned memory allocations; the size of allocated memory can be increased or decreased as per the requirements.

Now, suppose that the Client $\mathrm{Y}$ is unsatisfied with the service of the Service Provider B and wants to switch to the Service Provider A. 


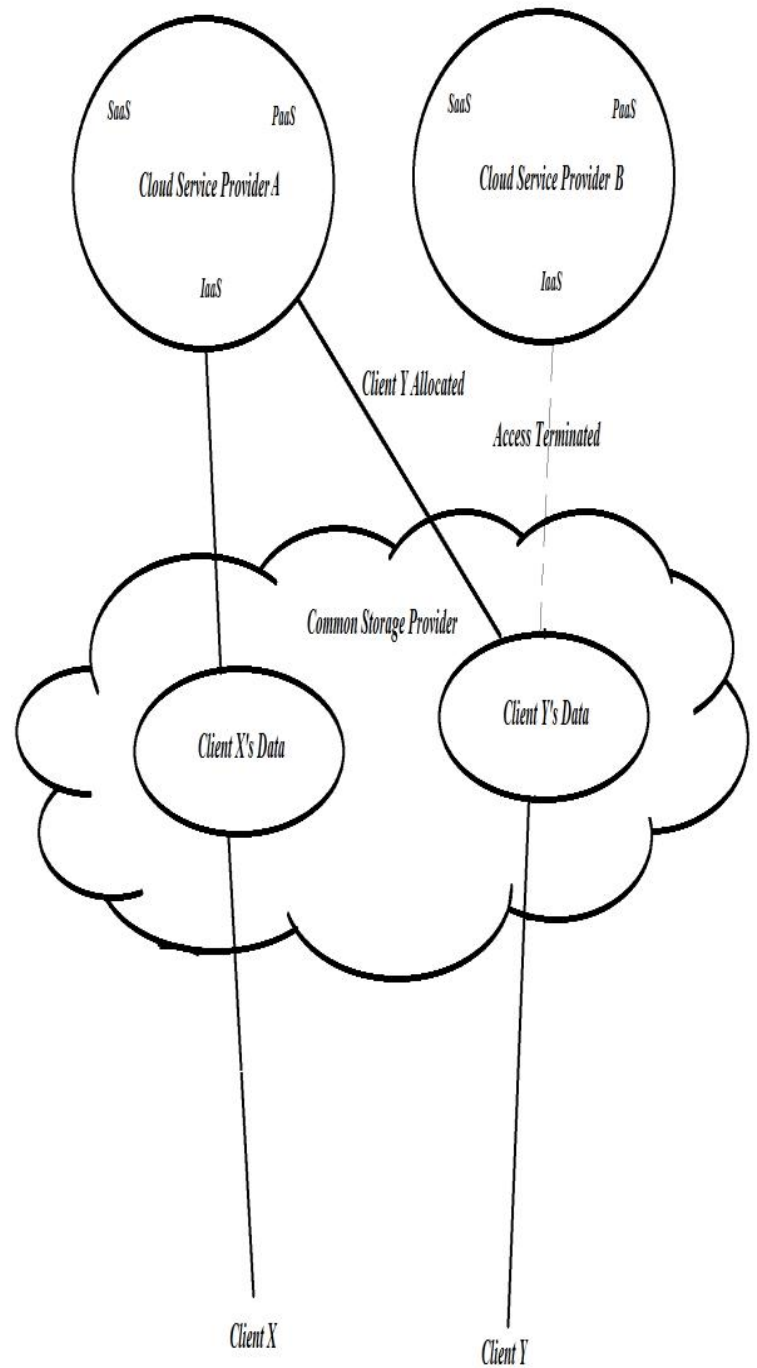

Fig 4: Switching from one service provider to another

To switch to the Service Provider A, Client Y first of all needs to inform the storage provider. The storage provider than terminates the Service Provider B's access to the data of Client Y. After that, storage provider (i.e. reliable third party) makes all the legal proceedings and settles all the payments due between the client $\mathrm{Y}$ and service provider $\mathrm{B}$. Once all the dues are made, the Client $\mathrm{Y}$ proceeds with legal formalities with the new service provider (i.e. A). Once these are completed, the portion of the storage provider's system having Client Y's data is simply allocated to the Service Provider A for further processing and management, thus removing the switching overhead costs and time.

\subsubsection{Cloud Consumer Forums:}

Whenever a cloud service consumer terminates the agreement or the issue of material breach is raised, there is a need of legal framework to settle the disputes, all the dues paid or to be paid. Until any decision is made by legal authority; the data is held by the service provider, making it unavailable to the concerned consumer (organization). Thus, due to any delay in the legal proceedings the organization suffers from great loss.

In order to give quick decisions on cloud service portability cases and other related issues, separate cloud consumer forums should be set up. The primary goal of the cloud consumer forums is to hear the disputes related to cloud portability issues and to give the decisions as quickly as possible. Other than that cloud consumer forums can also provide clients with best suitable service level agreements and can make them aware of any extensive disclaimers used by the service providers. Also the, short term cloud service contracts may provide more flexibility to meet the challenges of the changing environment [8].

\section{CONCLUSION}

The cloud services have provided new shape to the IT services but the lack of portability is the major critic preventing its wider adaptation. To make the cloud services portable, primarily the service providers should agree upon the use of the standardized and best suitable data formats and discard the usage of closed and proprietary data formats.

Secondly, it is required to secure the consumers data from getting locked in by service providers during termination of agreements. The introduction of the reliable third party between the cloud service provider and the service consumer removes all such issues and ensures the customer that his data is in safe hands.

The introduction of common storage provider also eliminates the switching time and cost, making the portability simpler and faster. Thus, it is the ultimate way of boosting the cloud service portability.

\section{REFERENCES}

[1] Robert H. Carpenter Jr. Walking from cloud to cloud: The portability issue in cloud computing. 6 WASH. J.L. $\begin{array}{llll}\text { TECH. } & \text { \& } & \text { ARTS } & 1\end{array}$ https://digital.lib.washington.edu/dspacelaw/handle/1773.1/447

[2] NIST Definition of Cloud Computing v15 http://csrc.nist.gov/groups/SNS/cloud-computing/

[3] Urs Gasser and John Palfrey. Mapping Cloud Interoperability in the Globalized Economy: Theory and Observations from Practice. NCCR trade regulation swiss national center of competence in research, June 2012.

[4] Communication From The Commission To The European Parliament, The Council, The European Economic And Social Committee And The Committee Of The Regions. Unleashing the Potential of Cloud Computing in Europe. Brussels, 27.9.2012 COM(2012) 529 final

[5] Apple says "Uncle," Carpenter Law Office Client Newsletter (Robert H. Carpenter, Jr., Plano, Tex.) Jan. Feb 2007 , available http://0093d40.netsolhost.com/images/ Apple_Says_Uncle_Jan._-_Feb_2007_2007.pdf 
[6] M. Behrendt et al., "IBM Cloud Computing Reference Architecture”, Open Group submission, Feb. 2011.

[7] Topology and Orchestration Specification of Cloud Applications (TOSCA), OASIS specification, Oct. 2011; www.oasis-

open.org/committees/tc_home.php?wg_abbrev= tosca.

[8] Office of Thrift Supervision, Thrift Bulletin 82A, Third Party Arrangements 15 (2004), available at http://www.ots.treas. Gov/_files/84272.pdf 